\title{
ASSESSMENT OF LOCAL MUSCULAR LOAD OF DENTAL PRACTITIONERS
}

\author{
Anna Schlenker ${ }^{1}$, Martin Kapitán², Lenka Vavřičková2, Milena Bušová1 \\ ${ }^{1}$ Institute of Hygiene and Epidemiology, First Faculty of Medicine, Charles University and General University Hospital in Prague, Prague, \\ Czech Republic \\ 2Department of Dentistry, Faculty of Medicine in Hradec Králové, Charles University and University Hospital Hradec Králové, Hradec Králové, \\ Czech Republic
}

\section{SUMMARY}

Objective: Hands and forearms are one of the most common localisations of musculoskeletal disorders (MSDs) among dental practitioners. The aim of this study was to objectively assess the local muscular load of hands and forearms of dental practitioners during various treatment procedures using the method of the integrated electromyography (iEMG). This method is used for health risk assessment and categorization of working operation within the official national methodology.

Methods: A total of 24 measurements were performed on 10 dental practitioners during 8 different dental treatments; mostly on those which are most frequent in clinical practice, i.e. endodontic treatment, tooth extraction, tooth restoration with filling and prosthetic treatment. The EMG Holter was used to detect the electromyographic potentials determining the local muscular load.

Results: All the muscle groups of the forearm were relatively evenly loaded at work. During the dynamic work activity, the average time-weighted value of maximum voluntary contraction (\%MVC) was in the range from 1 to $30 \% \mathrm{MVC}$ for all evaluated muscle groups. The mean average timeweighted value of \%MVC did not exceed $6 \%$ (a critical limit of the mean average time-weighted value of \%MVC) in any of the evaluated muscle groups. The results of the frequency analysis showed that large (55-70 \%MVC) and rarely the limits exceeding values (above $70 \% \mathrm{MVC}$ ) were observed for individual muscle groups. These forces are related to performing tasks at inaccessible locations associated with non-physiological working positions. The differences in the mean forearm muscle load between the upper limbs were statistically significant in total $(p<0.001)$, for flexors $(p=0.017)$ and for extensors $(p=0.006)$.

Conclusion: In view of the results of this study, the work of dentists can be ranked in category 2 in terms of the local muscle load factor according to the currently valid legislation in the Czech Republic.

Key words: integrated electromyography, local muscular load, evaluation, dental treatment, prevention of repetitive strain injury

Address for correspondence: A. Schlenker, Institute of Hygiene and Epidemiology, First Faculty of Medicine, Charles University and General University Hospital in Prague, Studničkova 7, 12800 Prague 2, Czech Republic. E-mail: schlenker.anna@gmail.com

https://doi.org/10.21101/cejph.a6157

\section{INTRODUCTION}

The incidence of occupational diseases due to the long-term excessive unilateral load of the upper extremities is increasing in the Czech Republic. The frequent cause is the local muscular load, which means the long-term excessive burden on small muscle groups of the forearm and hand.

The integrated electromyography (iEMG) is usually used for the evaluation of this burden. Integration is a mathematical process that calculates the area circumscribed by the curve. For the integration of EMG signals, a full-wave rectifier and an electronic integrator are used. Integrated electromyogram represents the total muscle activity and it is a function of the amplitude, duration and frequency of the course of each EMG potential. For measurements, a portable eight-channel polygraph is used to record physiological parameters, the EMG Holter with 4 EMG modules. EMG module is used to track muscle activity by measuring and recording the electrical potentials accompanying the muscle activity. EMG potentials are scanned by special surface electrodes.
One group of workers who may be affected by this type of load are dentists. The prevalence of work-related musculoskeletal disorders (WRMSDs) among dental practitioners ranges between $64 \%$ and $93 \%(1-5)$. One of the frequent locations of these disorders is the area of hands and forearms (6). The risk factors of the dental work, in terms of local muscular load, are high numbers of repeated unilateral movements in a constrained pace, the forced working position, applying high finger forces in some tasks, unfavourable working postures of the upper limbs, and the work expending big muscular forces (7). During different treatment procedures, the dentist loads various muscle groups according to the nature of the treatment. The cone beam computed tomography (CBCT) is an X-ray examination method producing three-dimensional images of the teeth and splanchnocranium. A wider spreading of its use is limited by a relatively high price of the machine; however, it is expected to be used more and more in the future.

The evaluation of the local muscular load is based on the limits set by Government Decree no. 361/2007 Coll., as amended, 
establishing conditions of health protection at work. We evaluate the average time-weighted value of maximum voluntary contraction $(\% \mathrm{MVC})$, the number of expended large muscle forces in the range of $55-70 \% \mathrm{MVC}$, the incidence of the over-limit muscle forces above $70 \% \mathrm{MVC}$, and the whole shift number of unilateral repetitive movements of hands and forearms with regard to expended forces. Categorization of working operations is the official national methodology for health risk assessment of working operations at workplaces in all occupations.

The evaluation of movements and ergonomics during work can be done in different ways:

- The method of in-situ observation - takes place several times for a limited period of time in order to determine the minute movements of the hands and forearms. The dentist is under the direct supervision of the evaluating person, which can be stressful for him/her.

- Analysis of the occupational activities from the video recording - is carried out in a similar way as the method of in-situ observation, i.e. a few times for a limited period of time to determine the number of minute movements of the hands and forearms. The benefits of this method are that it might be less stressful for the dentist and the evaluating person can better calculate the movements because of the possibility of slowing down or returning the record. A big advantage is also adding tags to recording and pairing video with integrated electromyography.

Our study was designed and planned with the aim to evaluate the local muscular load of hand and forearm muscles, the potential

Table 1. Selected dental treatments and quantity of measurements

\begin{tabular}{|l|c|}
\hline Dental treatment & Quantity \\
\hline Root canal treatment & 5 \\
\hline Prosthodontic treatment & 5 \\
\hline Tooth restoration & 4 \\
\hline Intra-alveolar extraction & 4 \\
\hline Transalveolar extraction & 3 \\
\hline Scaling and root planning & 1 \\
\hline Preventive check-up & 1 \\
\hline CBCT examination & 1 \\
\hline
\end{tabular}

CBCT - cone beam computed tomography risk of exceeding the limit values of \%MVC and the limit for movements during the dentists working shift.

\section{MATERIALS AND METHODS}

The study was performed on 10 dental practitioners, employees of the Department of Dentistry, Charles University, Faculty of Medicine in Hradec Králové, and University Hospital Hradec Králové, Czech Republic. A total of 24 measurements were performed during 8 types of dental treatment (Table 1). In the first phase, 8 different treatment modalities were selected and measured. In the second phase, the treatments with higher muscular load were measured more times. Table 2 presents short descriptions of four mostly measured treatments. The treated patients were previously booked for the planned dental procedure. Before the beginning of the treatment, they were asked to participate in this study and signed the informed consent. The inclusion into the study did not affect the selection of the treatment modality nor the process of the particular treatment. The dental practitioners participated voluntarily and signed informed consent. The study was approved by the Ethics Committee of the University Hospital Hradec Králové (Ref. no. 201803 S15P). The data was collected using the EMG Holter (GETA Centrum) to detect the electromyographic potentials determining the local muscular load (8).

The evaluating person observed the dental practitioner, checked the whole process of measuring and made notes about the specific characteristics of the dentist's work including the ergonomics. Individual measurements took 9 to 96 minutes depending on the type and extent of the treatment.

The dataset contains 24 measurements performed on 10 physicians during 8 different dental treatments. There were no errors and we did not need to exclude any record from further processing. Because of the diversity of the working procedures, we decided to split the results by performance.

The methods of descriptive statistics, Kolmogorov-Smirnov test and paired t-test were used for the statistical analysis. The level of statistical significance was set at $\alpha=0.05$.

\section{RESULTS}

The basic working position was sitting on a dental stool and/or standing, depending on the nature of the procedure performed. It was common to take the unacceptable and

Table 2. Short description of mostly measured treatments

\begin{tabular}{|l|l|}
\hline Dental treatment & Short description \\
\hline Root canal treatment & $\begin{array}{l}\text { Local anaesthesia, rubber dam placement, removal of fillings and caries, pre-endodontic build-up (if necessary), pulp chamber } \\
\text { roof removal, root canals negotiation, measurement, shaping, irrigation and obturation, and access cavity filling (permanent or } \\
\text { temporary). }\end{array}$ \\
\hline Prosthodontic treatment & $\begin{array}{l}\text { Local anaesthesia (if necessary), preparation of 2-6 teeth for crowns/fixed bridges, impressions, fabrication and luting of chairside } \\
\text { temporary crowns/bridges). }\end{array}$ \\
\hline Tooth restoration & Local anaesthesia, rubber dam placement, removal of fillings and caries, new composite filling placement. \\
\hline Intra-alveolar extraction & $\begin{array}{l}\text { Local anaesthesia, disruption of dentogingival attachment, 1-3 teeth loosening and extraction, socket excochleation, compresion, } \\
\text { gauze application. }\end{array}$ \\
\hline
\end{tabular}


conditionally acceptable trunk, head and upper limb working positions and work in a non-physiological trunk working position associated with its rotation. The recurrent occurrence of non-physiological working positions of the hand and wrist was noted, especially the wrist rotation and significant extension and flexion of the wrist and hand during interventions in difficult-to-reach places of the oral cavity. The dentists repeatedly used different types of tools. The character of the work was static-dynamic with a dynamic component predominating. All evaluated muscle groups of the forearms were relatively evenly loaded at work. The volume and content of work varied a lot in individual treatment procedures.

For the correct evaluation of workload during the dental treatment, the movements per minute of hands and forearms and average values of $\% \mathrm{MVC}$ were counted. The movements per minute were counted from a video record and the average values of \%MVC were measured directly by the electromyography software. The results are shown in Table 3.

The average time-weighted values of \%MVC for each work shift were from 1 to $30 \% \mathrm{MVC}$ for all assessed muscle groups.
Most of the probands in our study exceeded the valid hygienic limits for small repetitive movements, which is $6 \% \mathrm{MVC}$ according to the currently valid legislation in the Czech Republic. In some cases, it was more than three times (the values exceed $20 \% \mathrm{MVC}$ ).

The "root canal treatment" can be considered the most demanding type of treatment, where average values were around $20 \%$ MVC. However, the mean shift time-weighted value of $\% \mathrm{MVC}$ did not exceed $6 \% \mathrm{MVC}$ in any of the evaluated forearm muscle groups.

The frequency analysis showed that large (55-70\%MVC) and occasionally above-the-limit (over $70 \% \mathrm{MVC}$ ) muscular forces were repeatedly performed. The highest incidence of large exerted muscular forces was observed especially during the "intraalveolar tooth extraction". The occurrence of these forces is undoubtedly related to the engagement of forceful movements during performing tasks at inaccessible locations associated with work in the non-physiological working positions of the upper limbs and trunk.

Large muscle strengths in the range of 55-70\%MVC were mainly found during the prosthodontic treatment. The whole-shift number of large muscular forces exerted in the average work shift

Table 3. Measured average values of \%MVC for both right and left hand and forearm flexors and extensors during selected dental treatments

\begin{tabular}{|c|c|c|c|c|c|c|}
\hline \multirow{2}{*}{$\begin{array}{l}\text { Measurement } \\
\text { No. }\end{array}$} & \multirow[t]{2}{*}{ Dental treatment ${ }^{\star}$} & \multicolumn{2}{|c|}{$\begin{array}{c}\text { Average values of \%MVC for right } \\
\text { hand and forearm }\end{array}$} & \multicolumn{2}{|c|}{$\begin{array}{c}\text { Average values of \%MVC for left } \\
\text { hand and forearm }\end{array}$} & \multirow[t]{2}{*}{$\begin{array}{l}\text { Minute movements of hands } \\
\text { and forearms }\end{array}$} \\
\hline & & Flexors & Extensors & Flexors & Extensors & \\
\hline 2 & Root canal treatment & 4.02 & 16.45 & 22.23 & 23.29 & 122 \\
\hline 11 & Root canal treatment & 29.65 & 9.92 & 14.84 & 6.78 & 49 \\
\hline 16 & Root canal treatment & 6.75 & 20.55 & 1.34 & 0.98 & 138 \\
\hline 18 & Root canal treatment & 25.97 & 13.59 & 11.2 & 3.71 & 114 \\
\hline 24 & Root canal treatment & 22.96 & 9.63 & 12.94 & 4.41 & 113 \\
\hline 3 & Prosthodontic treatment & 13.93 & 15.96 & 8.87 & 7.23 & 121 \\
\hline 4 & Prosthodontic treatment & 13.13 & 18.37 & 7.44 & 7.95 & 115 \\
\hline 12 & Prosthodontic treatment & 22.71 & 11.11 & 20.55 & 19.87 & 105 \\
\hline 13 & Prosthodontic treatment & 24.10 & 11.36 & 18.90 & 9.43 & 74 \\
\hline 17 & Prosthodontic treatment & 15.52 & 11.15 & 11.72 & 5.79 & 114 \\
\hline 7 & Tooth restoration & 5.91 & 13.13 & 16.69 & 18.39 & 59 \\
\hline 15 & Tooth restoration & 19.67 & 17.20 & 13.88 & 9.77 & 64 \\
\hline 19 & Tooth restoration & 8.70 & 16.67 & 1.75 & 0.97 & 57 \\
\hline 20 & Tooth restoration & 23.12 & 10.01 & 13.38 & 6.84 & 97 \\
\hline 5 & Intra-alveolar extraction & 9.56 & 7.13 & 12.62 & 17.97 & 47 \\
\hline 6 & Intra-alveolar extraction & 8.17 & 10.81 & 15.92 & 22.62 & 46 \\
\hline 22 & Intra-alveolar extraction & 18.07 & 11.41 & 23.40 & 12.85 & 47 \\
\hline 23 & Intra-alveolar extraction & 15.64 & 9.39 & 16.24 & 7.71 & 55 \\
\hline 1 & Transalveolar extraction & 20.04 & 16.45 & 14.14 & 16.55 & 112 \\
\hline 10 & Transalveolar extraction & 14.80 & 11.79 & 13.38 & 17.26 & 78 \\
\hline 21 & Transalveolar extraction & 3.94 & 3.61 & 9.50 & 14.62 & 102 \\
\hline 8 & Scaling and root planning & 9.21 & 16.69 & 18.54 & 19.12 & 118 \\
\hline 14 & Preventive check-up & 8.79 & 8.39 & 0.81 & 0.71 & 66 \\
\hline 9 & CBCT examination & 14.08 & 27.79 & 1.75 & 0.88 & 127 \\
\hline
\end{tabular}

*The arrangement of measurement groups by the same type of dental treatment in the same order as in Table 1.

MVC - maximum voluntary contraction; CBCT - cone beam computed tomography 


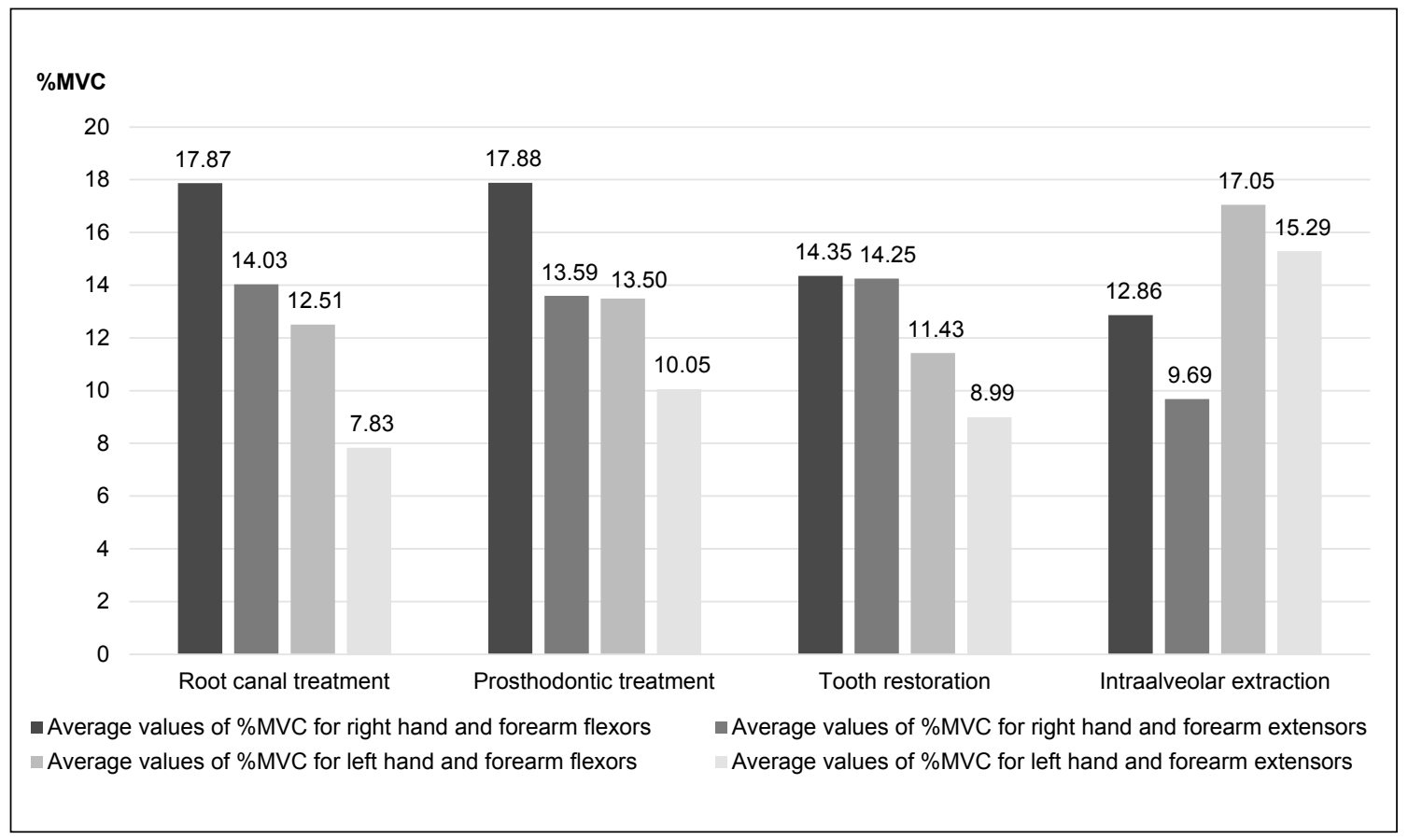

Fig. 1. Means of average values of $\% M V C$ for the more times measured treatments.

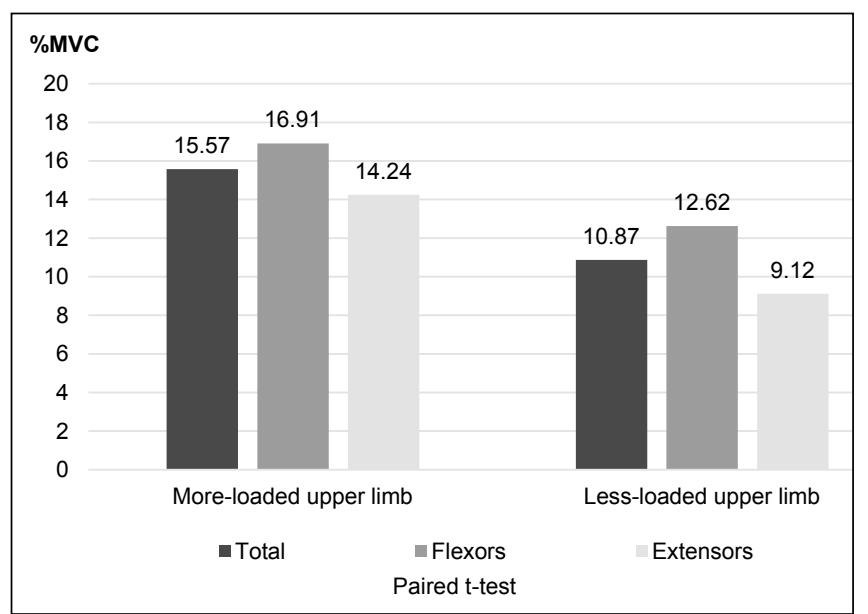

Fig. 2. Comparison of mean forearm muscle load between the more-loaded and less-loaded upper limbs.

in the standard work schedule does not exceed the applicable physiological limit.

The occurrence of muscle force above $70 \% \mathrm{MVC}$ has been reported only sporadically and its occurrence was mostly not related to structural work activity. The number of movements did not exceed the physiological limits in force in any of the work activities with respect to the muscular effort.

The means of the average values of $\% \mathrm{MVC}$ for the treatments measured more times ( 4 or 5 ) are shown in Figure 1. The differences in the mean forearm muscle load between the more-loaded and less-loaded upper limb were statistically significant in total $(p<0.001)$ and separately for flexors $(p=0.017)$ and for extensors $(\mathrm{p}=0.006)$. This comparison is demonstrated in Figure 2. In case of the root canal treatment, prosthodontic treatment and tooth restoration, the more-loaded upper limb was the right one; in case of the intra-alveolar extraction, the more-loaded one was the left upper limb.

\section{DISCUSSION}

The iEMG method was used in this study to evaluate the local muscular load of forearms of dental practitioners during work. It is currently the most accurate method available to monitor the response of the neuromuscular system to workload in the measured workers (9).

The occupation of dentists is one with a high prevalence of WRMSDs. As one of the frequent areas of complaints are upper limbs (7) we chose the measurement of the forearms' muscles. The dentists often report decreased muscle strength in hands and forearms. Our results, however, showed that the over-limit muscle forces (over 70\% of MVC) were rare and most of the probands in our study did not exceed the valid hygienic limits. In some cases, the average values exceed $20 \% \mathrm{MVC}$, but we can classify them as occasional.

The initially selected treatments are commonly and routinely performed by general dental practitioners. An exception is the CBCT examination of the patient, which is quite new diagnostic methods, but with a perspective of wider spreading in the future. Also, transalveolar extraction is not performed by all dental practitioners. It was included because we expected higher muscle loading caused by the nature and length of this treatment.

The treatment modalities with higher muscle load were measured more times in the second phase in different patients and different physicians to eliminate possible bias caused by the physician, patient and situation variability. In all evaluated types of treatment, the flexors were loaded more than extensors 
on both hands. This results from the nature of dental treatment when the dentist is holding a tool during the whole procedure. The held tool can be a working hand instrument or a handpiece in the dominant hand and a mirror in the other hand. Comparing the sides, the right hand was more loaded than the left hand in root canal treatment, prosthodontic treatment and tooth restoration. Surprisingly, during the intra-alveolar extraction, the left hand, i.e. not dominant, was more loaded than the right hand. The left hand is employed with the manual retraction of the surrounding soft tissues, keeping a good overview of the working field, and withholding the alveolar process around the extracted tooth. In hardly accessible areas this may be more physically demanding than the extraction itself, which is done using ergonomically suitable instruments by the dominant hand. All the dentists who carried out the four selected and further analysed treatment modalities were right-handed. The difference in the local muscle loading between both upper limbs was statistically significant, both in total and separately for flexors and for extensors. The overall data for all types of treatments together were used for this comparison. There was too small number of measurements for individual treatment types not allowing a relevant statistical analysis for different treatments separately. This finding confirms that the dental work is greatly unilateral and presents an asymmetrical loading to the dentists' upper limbs. Similar conclusions can be drawn from another study among dentists using the CUELA system (computer-assisted acquisition and long-term analysis of musculoskeletal loads) (10).

The results of our study correspond to our expectations. They can be compared, for example, to a previous study on the evaluation of local muscle load during typing (11), where it has also been shown that the forces were above the limit, but in an average shift did not exceed the hygienic limit.

It should be noted that the aim of our study was not assessing the workplace ergonomics and working posture of hands and forearms. We focused exclusively on just one of the important factors, namely the local muscular load during the selected dental treatments.

\section{CONCLUSION}

In view of the results of this study, we evaluated the potential risk of exceeding the limit values of \%MVC and the limit for movements during the dentists working shift. According to the currently valid legislation in the Czech Republic, the work of dentists can be ranked in category 2 in terms of the local muscle load factor.

The local muscular load measured on the forearms varied among different dental procedures. Most of the probands in this study exceeded the valid hygienic limit of $6 \% \mathrm{MVC}$ for small repetitive movements. The large muscular forces (55-70\% MVC) occurred repeatedly, whereas the above-the-limit forces (over $70 \% \mathrm{MVC}$ ) were seen occasionally. However, the mean shift time-weighted value of \%MVC did not exceed the limits.

The hands' and forearms' flexors were loaded more than extensors. The work of the dentists was asymmetrical with a significant difference in local muscle load of right and left upper limbs. During the root canal treatment, prosthodontic treatment and tooth restoration the dominant upper limb was more loaded, while during extraction the more loaded upper limb was the non-dominant one.

The incidence of occupational diseases due to the long-term excessive unilateral load of the upper extremities is increasing in the Czech Republic. The frequent cause is the local muscular load, which means the long-term excessive burden on small muscle groups of the forearm and hand.

In the future, we plan to enrich the study with an assessment of the ergonomics of the workplace and therefore create a comprehensive view of the workload of the dentist during shift-time. Furthermore, we plan to propose solutions to reduce the burden, e.g. by educating the worker on the use of appropriate worker positions, adherence to the workflow, work rotation, etc.

\section{Funding}

The study was financially supported by the Charles University programme PROGRESS Q25 and Q40/13.

\section{Acknowledgements}

The authors would like to thank the involved patients and dental practitioners for participation in this study.

\section{Conflict of Interests}

None declared

\section{REFERENCES}

1. Abbas SB, Qazi SR, Iftikhar S, Iqbal MU. Musculoskeletal disorders among dentists and dental students. Pak Oral Dental J. 2015;35(3):461-5.

2. Hayes MJ, Cockrell D, Smith DR. A systematic review of musculoskeletal disorders among dental professionals. Int J Dent Hyg. 2009;7(3):159-65.

3. Hodacova L, Sustova Z, Cermakova E, Kapitan M, Smejkalova J. Self-reported risk factors related to the most frequent musculoskeletal complaints among Czech dentists. Ind Health. 2015;53(1):48-55.

4. Leggat PA, Kedjarune U, Smith DR. Occupational health problems in modern dentistry: a review. Ind Health. 2007;45(5):611-21.

5. Šustová Z, Hodačová L, Kapitán M. The prevalence of musculoskeletal disorders among dentists in the Czech Republic. Acta Medica (Hradec Kralove). 2013;56(4):150-6.

6. Lindfors P, von Thiele U, Lundberg U. Work characteristics and upper extremity disorders in female dental health workers. J Occup Health. 2006;48(3):192-7.

7. Szymańska J. Disorders of the musculoskeletal system among dentists from the aspect of ergonomics and prophylaxis. Ann Agric Environ Med. 2002;9(2):169-73.

8. EMG Holter [Internet]. Prague: GETA Centrum s.r.o.; 2018 [cited 2020 Feb 14]. Available from: http://fyziologie.getacentrum.cz/emg-holter/. (In Czech.)

9. Hlávková J. Assessment of local muscle load [Internet]. Hradec Králové: Krajská hygienická stanice Královéhradeckého kraje se sídlem v Hradci Králové; 2016 [cited 2020 Feb 14]. Available from: http://www.khshk. cz/e-learning/kurs5/. (In Czech.)

10. Ohlendorf D, Erbe C, Hauck I, Nowak J, Hermanns I, Ditchen D, et al. Kinematic analysis of work-related musculoskeletal loading of trunk among dentists in Germany. BMC Musculoskel Dis. 2016;17(1):427. doi: 10.1186/s12891-016-1288-0.

11. Schlenker A, Tichý T. A New approach to the evaluation of local muscular load while typing on a keyboard. Cent Eur J Public Health. 2017;25(4):255-60. 